九州大学学術情報リポジトリ

Kyushu University Institutional Repository

\title{
The Combined Use of Runs in Statistical Quality Controls
}

Kitagawa, Toshio

Kyushu University

Seguchi, Tsunetami

Kyushu University

https://doi.org/10.5109/12975

出版情報: 統計数理研究. 7 (1/2)，pp. 25-45，1956-12. Research Association of Statistical Sciences

バージョン：

権利関係 : 


\title{
THE COMBINED USE OF RUNS IN STATISTICAL QUALITY CONTROLS
}

\author{
By \\ Tosio Kitagawa and Tsunetami SeguchI
}

(Received Oct. 25, 1956)

\$1. Introduction. The main aspects of the usual control chart method can be illustrated as follows. "The usual control chart controlling the mean of a population is constructed in the following way: After the mean and standard deviation of the population have been reliably estimated, samples of fixed size $n$ are selected and their arithmetic means $\bar{x}=\sum x / n$ are calculated. A chart is then constructed with control limits $m_{ \pm} B_{1} \sigma / \gamma / \bar{n}$, where $m$ and $\sigma$ are the estimates of the population mean and standard deviation, and $B_{1}=3$ or 3.09 . The various values $\bar{x}$ are entered in the chart in chronological order, and as soon as such values fall outside the control limits, production is stopped to allow investigation." (H. WeILER [4] p. 816817 ). In this connection it is to be noted that there are certain problems belonging to the realm of successive process of statistical inferences for estimating $m$ and / or $\sigma$ at the beginning of setting such control limits, as we have discussed in Kitagawa [2] Part III and [3] Part II. These fundamental aspects will be however out of consideration in what follows. Moreover in certain situations a set of alternative actions is prescribed one of which is to be taken according to the value $\bar{x}$, as particularly formulated in KITAgawa [3] Part II. These aspects will not be discussed in this paper. Now H. WEILER [4] investigates another aspect by introducing the following alternative control method. "Instead of stopping the production when a single $\bar{x}$ value falls outside the control limits $m_{ \pm} B_{1} \sigma / \sqrt{ } \bar{n}$, we may calculate the pair of narrower limits $m \pm B_{2} \sigma / v, \bar{n}$ and stop the production as soon as two successive $\bar{x}$ values fall above the upper or below the lower of these limits. More generally, we may calculate a pair of limits $m_{ \pm}$ $B_{\lambda} \sigma / V^{\prime} \bar{n}$ such that we may stop production as soon as $\lambda$ successive $\bar{x}$ values fall above the upper or below the lower of these limits. In each case, $B_{\lambda}$ is determined such that if the population mean does not change, an average of 1000 samples is necessary to produce one run of $\lambda$ successive $\bar{x}$ values above the upper (or below the lower) control limit." (H. WEILER [4] p. 817).

It is to be noticed that combined uses of some pairs of control limits are quite natural and often practically recommended, without involving any more costs and labours, while WEILER [4] discussed use of each one of these procedures separately. 
The object of this paper is to discuss with these combined uses of runs of several lengths with the usual control chart from the standpoint of recurrent probabilistic events in the sense of FELLER [1] Chapters 12, 13. In order to give the expected values of the numbers of trials attaining the first occurrence of the recurrent probabilistic events, we shall appeal to two Theorems, of which the first one Theorem 2.1 proved by KITAGAwA is concerned with direct use of functional equations, while the second one Theorem 3.1 proved by SEGUCHI with a decomposition of the event into a set of mutually disjoint recurrent probabilistic events.

Both of these two Theorems are indispensable for our present purpose. The Assumptions I and II to Theorem 2.1 may seem to be rather formal, but it will turn out to be fundamental one, as will be seen through more concrete examples given in the following paragraphes. In virtue of these two Theorems there is no difficulty at least in principle in developing some theory of combined uses of runs of several different lengths with the usual control charts. In $\S 4$ we shall treat with the simplest case of such combined used of runs of length 2 with the usual control chart, and in $\S 5$ we proceed to discuss with the more general case of uses of runs of lengths 2 and 3 with the usual chart method. Each of these two cases can be divided into several subclasses respectively, according to our rules of significance in our statistical decision procedures. It is to be noted that some of them $\mathbf{A}^{(+)}$(or $\mathbf{A}^{(-)}$) $\mathbf{A}^{( \pm)}, \mathbf{B}^{(+)}$(or $\mathbf{B}^{(-)}$) and $\mathbf{B}^{( \pm)}$may be suited for controlling any shift of the population mean $m$ to $\boldsymbol{m}+k \sigma$, while others $\mathrm{A}$ and $B$ suited for controlling any change of the population standard deviation $\sigma$ into $l \sigma$. The relative power associated with such combined uses of runs of one or several lengths with usual control chart method can be defined in term of the reciprocal of the expected value of the numbers of trials attaining the first occurrence of the recurrent probabilistic event.

Numerical results for our statistical procedures discussed in $\S 4$ and $\S 5$ are given in $\S 6$ with reference to a sequence of independent random samples of sizes 4 and 10 from a normal population of the type $N\left(m+k \sigma, \sigma^{2}\right)$, using tentatively or several control limits $B_{\lambda}$ given by WEILER [4]. In this paragraph $\$ 6$ we are not aiming to give efficient combinations of one or several control limits, but we are suggesting more practical situations to be taken in their considerations by quality control engineers when the uses of runs are proposed. For this purpose the controlling procedures $\mathbf{A}^{( \pm)}$ and $\mathbf{B}^{( \pm)}$are specially suited for the comparison with those of WEILER [4]. In $\$ 7$ the problem is discussed of how to set up control limits for our combined uses of runs of length 2 with the usual control chart method so as to satisfy assigned conditions on controlling powers.

$\$ 2$. The expected value of the numbers of trials attaining the first occurrence of a recurrent event associated with certain functional equations. 
Let $f(n)$ be the probability that the first occurrence of the recurrnt event $\varepsilon$ takes place at the $n$-th trial, where we adopt the definition of recurrent event according to FELLER [1] p. 240. Now let us make the following

Assumption I. There holds a functional equation

$$
f(n)=f(n-1)-\sum_{j=1}^{k+1} R_{j-1} f(n-j),
$$

for all positive integers $n \geqq k+2$, where $R_{\nu}(\nu=1,2, \cdots, k)$ are given nonnegative constants such that $R_{k}>0$. Let us define the generating function

$$
G(s) \equiv \sum_{n=1}^{\infty} s^{n} f(n)
$$

for a certain complex domain of $s$. For a while we shall be confined with a formal calculation, postponing the convergence problems of the allied power series as ones to be solved later according to specialised situations. The following Lemmas are quite immediate.

Lemma 2.1. Under the Assumption $I$, the generating function $G(s)$ can be written as

$$
L(s) G(s)=H(s) \text {, }
$$

where we have put

$$
\begin{gathered}
L(s) \equiv 1-s+\sum_{j=2}^{k+1} s^{j} R_{j-1} \\
H(s)=\sum_{\nu=1}^{k+1} s^{\nu} f(\nu)-s \sum_{\nu=1}^{k} s^{\nu} f(\nu)+\sum_{j=2}^{k+1} s^{j} R_{j-1}^{k-j+1} \sum_{\nu=1}^{\nu} f(\nu) .
\end{gathered}
$$

\section{Lemma 2.2. We have that}

$$
\begin{aligned}
H(s) & =\sum_{\nu=1}^{k-2} f(\nu)\left(s^{\nu}-s^{\nu+1}+\sum_{j=1}^{k-\nu-1} R_{j}\left(s^{\nu+j+1}-s^{k+1}\right)\right) \\
& +f(k-1)\left(s^{k-1}-s^{k}\right)+f(k)\left(s^{k}-s^{k+1}\right)+\left(\sum_{j=1}^{k} R_{j}\right)^{-k} s^{k+1},
\end{aligned}
$$

and that

$$
\begin{aligned}
& H^{\prime}(1)=(k+1) \sum_{j=1}^{k} R_{j}-\sum_{\nu=1}^{k-2} f(\nu)\left(1+\sum_{j=1}^{k-\nu-1}(k-\nu-j) R_{j}\right) \\
&-f(k-1)-f(k), \\
& L^{\prime}(1)=\sum_{j=1}^{k}(j+1) R_{j}-1 .
\end{aligned}
$$

The functional equation (2.01) gives us another functional euqation

$$
\begin{aligned}
f(n) & =\left(1-\sum_{\nu=1}^{n-2} f(\nu)\right) R_{1}+\left(1-\sum_{\nu=1}^{n-3} f(\nu)\right) R_{2}+\cdots \\
& +\left(1-\sum_{\nu=1}^{n-k} f(\nu)\right) R_{k-1}+\left(1-\sum_{\nu=1}^{n-k-1} f(\nu)\right) R_{k}
\end{aligned}
$$


for $n \geqq k+2$. Now it is necessary to have additional functional relations valid for $n \leqq k$. In view of the particular cases to be discussed in the following Sections, let us assume the following functional relations:

Assumption II. There is a positive constant $P_{0}$ less than one such that the relations hold for the sequence $\{f(j)\}(j=1,2, \cdots, k)$ to the effect that

$$
\begin{aligned}
f(j) & =\left(1-\sum_{\nu=1}^{j-2} f(\nu)\right) R_{1}+\left(1-\sum_{\nu=1}^{j-3} f(\nu)\right) R_{2}+\cdots \\
& +(1-f(1)) R_{j-2}+R_{j-1}+P_{0}^{-1} R_{j}
\end{aligned}
$$

for $j=3,4, \cdots, k$ and moreover that

$$
\begin{aligned}
& f(1)=P_{0}^{-1} R_{1}, \\
& f(2)=P_{0}^{-1} R_{1}+R_{2} .
\end{aligned}
$$

Lemma 2.3. Under the Assumptions $I$ and $I I$, we have

$$
H^{\prime}(1)=\sum_{j=1}^{k}(j+1) R_{j}-P_{0}^{-1} \sum_{j=1}^{k} R_{j} .
$$

Proof: (2.10) gives us, in particular for $j=k$, the relation

$$
f(k)=\sum_{j=1}^{k-1} R_{j}+P_{0}^{-1} R_{k}-\sum_{j=1}^{k-2}\left(\sum_{\nu=1}^{k-j-1} f(\nu)\right) R_{j},
$$

which, in combination with (2.07), yields us

$$
\begin{aligned}
H^{\prime}(1)= & k \sum_{j=1}^{k-1} R_{j}+(k+1) R_{k}-P_{0}^{-1} R_{k} \\
& -\sum_{v=1}^{k-3} f(\nu)\left(1+\sum_{j=1}^{k-\nu-1}(k-\nu-1-j) R_{j}\right) \\
& -f(k-2)-f(k-1) .
\end{aligned}
$$

The use of (2.10) for $j=k-1$ gives us

$$
f(k-1)=\sum_{j=2}^{k-2} R_{j}+P_{0}^{-1} R_{k-1}-\sum_{j=1}^{k-3}\left(\sum_{\nu=1}^{k-j-2} f(\nu)\right) R_{j},
$$

which, in combination of (2.15), yields us

$$
\begin{aligned}
H^{\prime}(1) & =(k-1) \sum_{j=1}^{k-2} R_{j}+k R_{k-1}+(k+1) R_{k}-P_{v}^{-1} R_{k-1}-P_{v}^{-1} R_{k} \\
& -\sum_{\nu=1}^{k-1} f(\nu)\left(1+\sum_{j=1}^{k-1-3}(k-\nu-2-j) R_{j}\right)-f(k-3)-f(k-2) .
\end{aligned}
$$

Now this procedure can be repeated by the use of the relation (2.10) to the consequence that

$$
H^{\prime}(1)=\sum_{j=3}^{k+1}(j+1) R_{j}+3\left(R_{2}+R_{1}\right)-P_{0}^{-1} \sum_{j=3}^{k+1} R_{j}-f(1)-f(2) .
$$

But in virtue of the relations (2.11) and (2.12) we have the relation (2.13) as was to be proved. 
Theorem 2.1. Under the Assumptions $I$ and $I I$, the recurrent probabilistic event $\varepsilon$ is certain and the mean recurrence time of $\varepsilon$ can be expressed by virtue of the constants $P_{0}, R_{1}, R_{2}, \cdots, R_{k-1}$ and $R_{k}$ such that

$$
\begin{gathered}
S \equiv \sum_{j=1}^{\infty} f(j)=1, \\
T \equiv \sum_{j=1}^{\infty} j f(j)=\frac{1-P_{0}^{-1} \sum_{j=1}^{k} R_{j}}{\sum_{j=1}^{k} R_{j}} .
\end{gathered}
$$

Proof : Since $L(1)=H(1)=R_{1}+R_{2}+\cdots+R_{k}$, we have

$$
\begin{gathered}
S \equiv G(1)=1, \\
T=G^{\prime}(1)=\left(H^{\prime}(1)-L^{\prime}(1)\right) / L(1) .
\end{gathered}
$$

Now (2.20) is immediate from Lemmas 2.2 and 2.3.

\$. The relation between the expected values of number of trials attaining the first occurrence of mutually disjoint recurrent events.

Let $\varepsilon_{i}(i=1,2, \cdots, k)$ be $k$ recurrent probabilistic events in the sense of FELLER [1], and let us denote by $f_{i}(n)$ the probability that $\varepsilon_{i}$ occurs for the first time at trial number $n$, by $u_{i}(n)$ the probability that $\varepsilon_{i}$ occurs at the $n$-th trial (not necessarily for the first time) for $i=1,2, \cdots, k$. Let us write

$$
\begin{aligned}
& U_{l}(s)=\sum_{n=1}^{\infty} u_{i}(n) s^{n}, \\
& F_{l}(s)=\sum_{n=1}^{\infty} f_{l}(n) s^{n},
\end{aligned}
$$

for $i=1,2, \cdots, k$.

Now by the joint event $\varepsilon \equiv \varepsilon_{1} \bigcup \varepsilon_{2} \bigcup \cdots \cup \varepsilon_{k}$ we mean the event which occurs when and only when at least one of $k$ events occurs. In view of the definition of recurrent event [FELLER [1] p. 240, Definition], the joint event is also a recurrent probabilistic event, and let us denote by $f(n)$ the probability that the joint event $\varepsilon$ occurs for the first time at trial number $n$, by $u(n)$ the probability that the joint event occurs at the $n$-th trial (not necessarily for the first time). Let us write

$$
\begin{aligned}
& U(s)=\sum_{n=0}^{\infty} u(n) s^{n}, \\
& F(s)=\sum_{n=1}^{\infty} f(n) s^{n} .
\end{aligned}
$$

For the sake of the convenience, we, shall assume $u_{\imath}(0)=u(0)=1$.

Definition 3.1. $k$ recurrent probabilistic events $\left\{\varepsilon_{i}\right\}(i=1,2, \cdots, k)$ are called to be mutually disjoint, if we have 


$$
u(n)=u_{1}(n)+u_{2}(n)+\cdots+u_{k}(n)
$$

for all positive integers $n$.

Theorem 3.1. Let $\left\{\varepsilon_{i}\right\}(i=1,2, \cdots, k)$ be $k$ mutually disjoint recurrent probabilistic events each of which is certain. Let $T_{1}(i=1,2, \cdots, k)$ and $T$ be the expected values of numbers of trials attaining the first occurrences of $\varepsilon_{1}(i=1,2, \cdots, k)$ and the joint event $\varepsilon$ respectively. Let all of $T_{1}$ be different from zero. Then we have

$$
\frac{1}{T}=\frac{1}{T_{1}}+\frac{1}{T_{2}}+\cdots+\frac{1}{T_{k}} .
$$

Proof: In view of the relation (3.05) and the assumption that $u_{i}(0)$ $=u(0)=1(i=1,2, \cdots, k)$, we have

$$
U(s)=U_{1}(s)+U_{2}(s)+\cdots+U_{k}(s)-(k-1) .
$$

Now in virtue of the relation

$$
\left(1-F_{i}(s)\right) U_{i}(s)=(1-F(s)) U(s)=1,
$$

we have, after differentiation,

$$
\frac{F^{\prime}(s)}{(1-F(s))^{2}}=\sum_{i=1}^{k} \frac{F_{i}^{\prime}(s)}{\left(1-\bar{F}_{i}(s)\right)^{2}} .
$$

But we have

$$
\begin{gathered}
T=F^{\prime}(1), \quad T_{i}=F_{i}^{\prime}(1), \quad(i=1,2, \cdots, k), \\
F(1)=F_{\imath}(1)=F_{:}(1)=\cdots=F_{k}(1)=1,
\end{gathered}
$$

because $\varepsilon_{\mathfrak{l}}$ and $\varepsilon$ are certain. : Moreover we note that

$$
\lim _{s \rightarrow 1}\left(\frac{1-F(s)}{1-F_{i}(s)}\right)^{2}=\left(\frac{F^{\prime}(1)}{F_{\imath}(1)}\right)^{2} .
$$

The limit of (3.09) as $s$ tends to 1 gives us, in view of (3.12), the relation (3.06), as it was to be proved.

\$4. Statistical controlling methods associated with the combined use of the runs of length 2 with the usual control chart method. Let $a_{1}$ and $a_{2}$ be prescribed real numbers such that $0 \leqq a_{2} \leqq a_{1}<\infty$. The set of all real numbers $-\infty<y<\infty$ is divided into the mutually exclusive sets $E_{ \pm 0}, E_{ \pm 1}$ and $E_{ \pm 2}$ such that

$$
E_{+0}=\left[y ; 0<y<a_{2}\right],
$$$$
E_{-0}=\left[y ;-a_{2}<y \leqq 0\right],
$$$$
E_{+1}=\left[y ; y \geqq a_{1}\right] \text {, }
$$$$
E_{-1}=\left[y ; y \leqq-a_{1}\right] \text {, }
$$$$
E_{+2}=\left[y ; a_{2} \leqq y<a_{1}\right] \text {, }
$$$$
E_{-2}=\left[y ;-a_{1}<y \leqq-a_{2}\right] \text {. }
$$

Let us write also

$$
E_{j}=E_{+} \cup E_{-j} \quad(j=0,1,2) .
$$


Let $\left\{Y_{i}\right\}(i=1,2,3, \cdots)$ be a sequence of independent stochastic variables each of which is distributed according to the same distribution. Let us denote three common probabilities for all $Y_{i}(i=1,2, \cdots)$ by
$\operatorname{Pr} .\left\{Y_{i} \in E_{ \pm j}\right\} \equiv P_{ \pm j}$
$(j=0,1,2)$,
$\operatorname{Pr} .\left\{Y_{i} \in E_{j}\right\} \equiv P_{j}=P_{+j}+P_{-j}$
$(j=0,1,2)$.

Four types of statistical controlling methods will now be introduced according to the ways how the significance of the sequence of $\left\{y_{b}\right\}$ will be defined.

[1] Statistical controlling method $\mathbf{C}(1,2)\left[a_{1}, a_{2}\right]$. Our statistical decision rule $\mathbf{A}$ is defined as follows: A sample $\left\{\boldsymbol{y}_{i}\right\}$ of the sequence $\left\{\boldsymbol{Y}_{\boldsymbol{i}}\right\}$ is said to be significant at the $n$-th step if either one of the following conditions is satisfied:

$$
\left(\mathrm{A}_{1}\right) y_{2} \in E_{1} ;\left(\mathrm{A}_{2}\right) y_{n-1} \in E_{2} \text {, and } y_{n} \in E_{1} \cup E_{2} \text {. }
$$

It is to be noted that the two conditions $\left(A_{1}\right)$ and $\left(A_{2}\right)$ are not mutually exclusive. Indeed for instance the case when $\left(\mathrm{A}_{3}\right) y_{n-1} \in E_{2}$ and $y_{n} \in E_{1}$ is a special case of both $\left(\mathrm{A}_{1}\right)$ and $\left(\mathrm{A}_{2}\right)$. A sample $\left\{y_{i}\right\}(i=1,2, \cdots, n)$ of the sequence $\left\{Y_{i}\right\}$ is said to be non-significant up to and including the $n$-th step, if there is no $j$ in $1 \leqq j \leqq n$ at whose step $\left\{y_{i}\right\}(1 \leqq i \leqq j)$ is significant. A sample $\left\{y_{i}\right\}$ of the sequence $\left\{Y_{i}\right\}$ is said to be significant for the first time at the $n$-th step, if it is not significant up to and including the $(n-1)$-th step and it is significant just at the $n$-th step. Let us define the probabilistic event $\varepsilon_{A}$ which occurs when and only when the sequence $\left\{Y_{i}\right\}$ becomes significnat. Let $f_{A}(n)$ be the probability that the sequence $\left\{Y_{t}\right\}$ becomes significant, that is, $\varepsilon_{\mathrm{A}}$ occurs, at the $n$-th step for the first time in this rule $\mathbf{A}$. Now it is evident that $\varepsilon_{\mathrm{A}}$ is a recurrent probabilistic event as a special case of that defined in $\$ 2$. Defining $k=2$ and

$$
R_{1}(\mathrm{~A})=P_{0} P_{1}, \quad R_{1}(\mathrm{~A})=P_{0} P_{2}\left(P_{1}+P_{2}\right),
$$

we have the functional equation for $n \geqq 4$

$$
f_{\mathrm{A}}(n)-f_{\mathrm{A}}(n-1)+R_{1}(\mathrm{~A}) f_{\mathrm{A}}(n-2)+R_{2}(\mathrm{~A}) f_{\mathrm{A}}(n-3)=0,
$$

with the additional condtions

$$
\begin{aligned}
f_{\mathrm{A}}(1) & =R_{1}(\mathrm{~A}) P_{0}^{-1}, \\
f_{\mathrm{A}}(2) & =R_{1}(\mathrm{~A})+R_{2}(\mathrm{~A}) P_{0}^{-1}=P_{0} P_{1}+P_{2}\left(P_{1}+P_{2}\right), \\
f_{\mathrm{A}}(3) & =\left(1-f_{\mathrm{A}}(1)\right) R_{1}(\mathrm{~A})+R_{2}(\mathrm{~A}) \\
& =\left(1-P_{1}\right) P_{0} P_{1}+P_{0} P_{2}\left(P_{1}+P_{2}\right) .
\end{aligned}
$$

Indeed the direct calculations give us $(4.061) \sim(4.063)$ in view of the relations : 
(4.071) $f_{A}(1)=\operatorname{Pr} .\left\{Y_{1} \in E_{1}\right\}$,

(4.072) $f_{A}(2)=\operatorname{Pr} .\left\{Y_{1} \in E_{v}\right\} \operatorname{Pr} .\left\{Y_{2} \in E_{1}\right\}+\operatorname{Pr} .\left\{Y_{1} \in E_{2}\right\} \operatorname{Pr} .\left\{Y_{2} \in E_{2} \cup E_{:}\right\}$,

(4.073) $f_{\mathrm{A}}(3)=\operatorname{Pr} .\left\{Y_{1} \bar{\mp} E_{1}\right\} \operatorname{Pr} .\left\{Y_{2} \in E_{v}\right\} \operatorname{Pr} .\left\{Y_{3} \in E_{1}\right\}$

$$
+\operatorname{Pr} .\left\{Y_{1} \in E_{0}\right\} \operatorname{Pr} .\left\{Y_{2} \in E_{2}\right\} \operatorname{Pr} .\left\{Y_{3} \in E_{1} \cup E_{2}\right\} \text {. }
$$

For $n \geqq 4$, the occurrences of the significance at the $n$-th step for the first instance are possible exactly in one of the following three exclusive ways :

(1.) The first case $W_{1}(\mathrm{~A})$. This means a simultaneous occurrence of the three events: (i) $\left\{y_{i}\right\}(i=1,2, \cdots, n-2)$ is non-significant; (ii) $y_{n-1}$ e $E_{0}$; (iii) $y_{n} \in E_{1}$.

$\left(2^{\circ}\right)$ The second case $W_{2}(\mathrm{~A})$. This means a simultaneous occurrence of the four events: (i) $\left\{y_{i}\right\}(i=1,2, \cdots, n-3)$ is non-significant; (ii) $y_{n-2}$ $\in E_{0}$; (iii) $y_{n-1} \in E_{2}$; (iv) $y_{n} \in E_{2}$.

$\left(3^{\circ}\right)$ The third case $W_{3}(\mathrm{~A})$. This means a simultaneous occurrence of the four events: (i) $\left\{y_{i}\right\}(i=1,2, \cdots, n-3)$ is non-significant; (ii) $y_{n-2} \in E_{0}$; (iii) $y_{n-1} \in E_{2}$; (iv) $y_{n} \in E_{1}$.

Now it can be readily seen that

$$
\operatorname{Pr} .\left\{W_{1}(\mathrm{~A})\right\}=\left(1-\sum_{i=1}^{n-2} f_{A}(i)\right) P_{0} P_{1},
$$

$$
\operatorname{Pr} .\left\{W_{2}(\mathrm{~A})+W_{3}(\mathrm{~A})\right\}=\left(1-\sum_{i=1}^{n-3} f_{\mathrm{A}}(i)\right) P_{\mathrm{v}}\left(P_{2}^{2}+P_{2} P_{1}\right) \text {. }
$$

Consequently we have, for $n \geqq 3$,

$$
\begin{aligned}
f_{\mathrm{A}}(n)- & \sum_{i=1}^{3} \operatorname{Pr} .\left\{W_{i}(\mathrm{~A})\right\}=\left(1-\sum_{i=1}^{n-2} f_{\mathrm{A}}(i)\right) P_{0} P_{1} \\
& +\left(1-\sum_{i=1}^{n-3} f_{\mathrm{A}}(i)\right) P_{0} P_{2}\left(P_{1}+P_{i 2}\right) \\
& =\left(1-\sum_{i=1}^{n-2} f_{\mathrm{A}}(i)\right) R_{1}(\mathrm{~A})+\left(1-\sum_{i=1}^{n-3} f_{\mathrm{A}}(i)\right) R_{2}(\mathrm{~A}),
\end{aligned}
$$

which will lead us to (4.05), since we have for all $n \geqq 4$,

$$
f_{A}(n-1)=\left(1-\sum_{i=1}^{n-3} f_{\mathrm{A}}(i)\right) R_{1}(\mathrm{~A})+\left(1-\sum_{i=1}^{n-4} f_{\mathrm{A}}(i)\right) R_{2}(\mathrm{~A}) \text {. }
$$

The controlling method associated with this statistical decision rule A may be called as the control chart method of the combined type $\mathbf{C}(1,2)$, while the single use of the condition $\left(A_{1}\right)$ corresponds to the usual control chart $\mathbf{C}(1)$ and that of the condition $\left(A_{2}\right)$ to the single use of run of length 2 , $\mathbf{C}(2)$ in the sense different from that of WeILER [4]. The corresponding expected values of the numbers of trials attaining their first occurrence of significance will be denoted $T(1,2), T(1)$ and $T(2)$ respectively. Now we observe 
Theorem 4.1. For the statistical decision rule $\mathbf{A}$, the probability that our sequence $\left\{Y_{i}\right.$ i becomes significant is certain, that $i s$,

$$
S(1,2) \equiv \sum_{n=1}^{\infty} f(n)=1 \text {, }
$$

and moreover the relation holds

$$
T(1,2) \equiv \sum_{n=1}^{\infty} n f(n)=\frac{1}{P_{1}+\ldots P_{1}^{2}}=\frac{1}{1+P_{2}^{-}}=\frac{1}{T(1)}+\frac{1}{T(2)} .
$$

Proof: The proof is immediate from Theorem 2.1.

[2] Statistical controlling methods $\mathbf{C}^{(+)}(1,2), \mathbf{C}^{(-)}(1,2)$ and $\mathbf{C}^{( \pm)}(1,2)$. Let us define the two statistical decision rules $\mathbf{A}^{(+)}$and $\mathbf{A}^{(-)}$similarly to the statistical decision rules $A$ by replacing the conditions $(3.03)\left(A_{1}\right)$ and $\left(\mathrm{A}_{2}\right)$ by

$$
\left(\mathrm{A}_{1}^{(+)}\right) y_{n} \in E_{+1} ; \quad\left(\mathrm{A}_{2}^{(+)}\right) y_{n-1} \in E_{+2} \text { and } y_{n} \in E_{+1} \cup E_{+2}
$$

for the rule $A^{(+)}$, and by

$$
\left(\mathrm{A}_{1}^{(-)}\right) y_{n} \in E_{-1} ; \quad\left(\mathrm{A}_{-2}^{(-)}\right) y_{n-1} \in E_{-2} \text { and } y_{n} \in E_{-1} \cup E_{-2}
$$

for the rule $\mathbf{A}^{(-)}$respectively. Let us define the probabilistic events $\varepsilon_{\mathrm{A}(+)}$ which occurs when and only when $\left\{Y_{i}\right\}$ becomes significant according to (4.13), and similarly $\varepsilon_{\mathrm{A}}$, ) according to (4.14). Moreover let us define the probabilistic event $\varepsilon_{\mathrm{A}( \pm)}=\varepsilon_{\mathrm{A}(+)} \bigcup \varepsilon_{\mathrm{A}(-)}$ which occurs when and only when $\left\{Y_{i}\right\}$ becomes significant according either to (4.13) or to (4.14) and corresponding statistical decision rule $\mathbf{A}^{( \pm)}$.

The controlling methods allied to each of these statistical decision rules $\mathbf{A}^{(+)}, \mathbf{A}^{(-)}$and $\mathbf{A}^{( \pm)}$will be denoted by $\mathbf{C}^{(+)}(1,2)\left[a_{1}, a_{2}\right], \mathbf{C}^{(-)}(1,2)$ $\left[-a_{1},-a_{.}\right]$and $\mathbf{C}^{( \pm)}(1,2)\left[a_{1}, a_{2}\right]$ respectively.

Let us denote by $T^{(+)}(1,2), T^{(-i}(1,2)$ and $T^{( \pm)}(1,2)$ the expected value of numbers of trials attaining the first occurrence of significance in each of $\varepsilon_{\left.A^{(}+\right)}, \varepsilon_{\mathrm{A}^{(-)}}$and $\varepsilon_{\left.\mathrm{A}^{(} \pm\right)}$respectively, while the probabilities that our sequence $\left\{Y_{1}\right\}$ becomes significant in each of $\varepsilon_{\mathrm{A}}(+), \varepsilon_{\mathrm{A}(-)}$ and $\varepsilon_{\mathrm{A}}( \pm)$ by $S^{(\tau)}(1,2), S^{(-)}(1,2)$ and $S^{( \pm)}(1,2)$ respectively. We observe

Theorem 4.2. We have

$$
\begin{aligned}
& S^{(+)}(1,2)=S^{(-)}(1,2)=S^{( \pm)}(1,2)=1, \\
& T^{(+)}(1,2)=\frac{1}{P_{+1}+\frac{P_{+2}^{2}}{1+P_{+2}}}=\frac{1}{\frac{1}{T^{(+)}(1)}+\frac{1}{T^{(+)}(2)}}, \\
& T^{(-)}(1,2)=-\frac{1}{P_{-1}+\frac{1}{1+P_{-2}^{2}}}=\frac{1}{\frac{1}{T_{-2}^{(-)}(1)}+T^{(-)}(2)},
\end{aligned}
$$


and

$$
T^{( \pm)}(1,2)=\frac{1}{P_{1}+\frac{P_{+2}^{2}}{1+P_{+2}}+\frac{P_{-2}^{2}}{1+P_{-2}}}=\frac{1}{\frac{1}{T^{(+)}(1,2)}+\frac{1}{T^{(-)}(1,2)}}
$$

Proof: The event $\varepsilon_{\mathrm{A}(+)}$ is a recurrent probabilistic event as a special case of that defined in $\S 2$. Defining $k=2$ and

$$
\begin{aligned}
& R_{1}\left(\mathrm{~A}^{(+)}\right)=\left(P_{0}+P_{-2}+P_{-1}\right) P_{+1}, \\
& R_{2}\left(\mathrm{~A}^{(+)}\right)=\left(P_{0}+P_{-2}+P_{-1}\right)\left(P_{+1}+P_{+2}\right),
\end{aligned}
$$

we have the functional equation similar to (4.05) to be obtained by replacing $R_{i}(\mathrm{~A})$ by $R_{\imath}\left(\mathrm{A}^{(+)}\right)(i=1,2)$ respectively, with the additional conditions

$$
\begin{aligned}
& f_{\mathrm{A}(+)}(1)=R_{1}\left(\mathrm{~A}^{(+)}\right)\left(P_{0}+P_{-2}+P_{-1}\right)^{-1}, \\
& f_{\mathrm{A}(+)}(2)=R_{1}\left(\mathrm{~A}^{(+)}\right)+R_{2}\left(\mathrm{~A}^{(+)}\right)\left(P_{0}+P_{-2}+P_{-1}\right)^{-1}, \\
& f_{\mathrm{A}(+)}(3)=\left(1-P_{+1}\right) R_{1}\left(\mathrm{~A}^{(+)}\right)+R_{2}\left(\mathrm{~A}^{(+)}\right) .
\end{aligned}
$$

In view-of these facts, it is evident that we car obtain $S^{(+)}(1,2)=1$ and the formula (4.16) similarly as in the event $\varepsilon_{\mathrm{A}}$; it suffices us to replace $P_{0}, P_{1}$ and $P_{2}$ by $P_{0}+P_{-1}+P_{-2}, P_{+1}$ and $P_{+2}$ respectively.

Similar arguments hold true for $\epsilon_{\mathrm{A}(-)}$.

The remaining parts of (4.15) and (4.18) can be immediately obtained in virtue of Theorem 3.1.

\$5. Statistical controlling methods associated with the combined use of the runs of lengths 2 and 3 with the usual control chart method.

Let $a_{1}, a_{2}$, and $a_{3}$ be prescribed real numbers such that $0 \leqq a_{3} \leqq a_{2} \leqq$ $a_{1}<\infty$. The set of all real numbers $-\infty<y<\infty$ is divided into the mutual exclusive set $E_{ \pm 0}, E_{ \pm 1}, E_{ \pm 2}$ and $E_{ \pm 3}$ where

$$
\begin{array}{ll}
E_{+0}=\left[y ; 0<y<a_{3}\right], & E_{-0}=\left[y ;-a_{3}<y \leqq 0\right], \\
E_{+1}=\left[y ; y \geqq a_{1}\right], & E_{-2}=\left[y ; y \leqq-a_{1}\right], \\
E_{+2}=\left[y ; a_{2} \leqq y<a_{1}\right], & E_{-2}=\left[y ;-a_{1}<y \leqq-a_{3}\right], \\
E_{+3}=\left[y ; a_{3} \leqq y<a_{3}\right], & E_{-3}=\left[y ;-a_{2}<y \leqq-a_{3}\right] .
\end{array}
$$

Let us write also

$$
E_{\jmath}=E_{+} \cup E_{-j} \quad(j=0,1,2,3) .
$$

Let $\left\{Y_{i}\right\}(i=1,2,3, \cdots)$ be a sequence of independent stochastic variables each of which is distributed according to the same distribution. Let us denote three common probabilities for all $\left\{Y_{i}\right\}(i=1,2,3, \cdots)$ by 


$$
\begin{array}{ll}
\operatorname{Pr} .\left\{Y_{i} \in E_{ \pm j}\right\}=P_{ \pm j} & (j=0,1,2,3), \\
\operatorname{Pr} .\left\{Y_{\imath} \in E_{j}\right\}=P_{j}=P_{+j}+P_{-j} & (j=0,1,2,3) .
\end{array}
$$

[1] Statistical controlling method $\mathbf{C}(1,2,3)$. The statistical decision rule $\mathbf{B}$ is defined as follows: A sample $\left\{y_{i}\right\}$ of the sequence $\left\{Y_{1}\right\}$ is said to be significant at the $n$-th step if either one of the following situations happens:

$$
\begin{aligned}
& \text { (B) } y_{n} \in E_{1} ;\left(\mathrm{B}_{2}\right) y_{n-1} \in E_{2} \text { and } y_{n} \in E_{1} \cup E_{2} \\
& \left(\mathrm{~B}_{3}\right) y_{n-2} \in E_{3}, y_{n-1} \in E_{2} \cup E_{3} \text { and } y_{n} \in E_{1} \cup E_{2} \cup E_{3} ; \\
& \left(\mathrm{B}_{4}\right) y_{n-2}=E_{2}, y_{n-1} \in E_{3} \text { and } y_{n} \in E_{1} \cup E_{2} \cup E_{3} .
\end{aligned}
$$

It is to be noted that the three conditions $\left(B_{1}\right),\left(B_{2}\right),\left(B_{3}\right)$ and $\left(B_{1}\right)$ of $(5.03)$ are not mutually exclusive. A sample $\left\{y_{i}\right\}(i=1,2, \cdots, n)$ of the sequence $\left\{Y_{i}\right\}$ is said to be non-significant up to and including the $n$-th step if there is no $j$ in $1 \leqq j \leqq n$ at whose step $\left\{y_{b}\right\} 1 \leqq i \leqq j$ is significant. A sample $\left\{y_{b}\right\}$ of the sequence $\left\{Y_{t}\right\}$ is said to be significant for the first time at the $n$-th step if it is not significant up to and including the $(n-1)$-th step and it is significant just at the $n$-th step.

Let us define the probabilistic event $\varepsilon_{\mathrm{B}}$ which occurs when and only when the sequence $\left\{Y_{i}\right\}$ becomes significant. Let $f_{\mathrm{B}}(\boldsymbol{n})$ be the probability that the sequence $\left\{Y_{1}\right\}$ becomes significant, that is, $\varepsilon_{13}$ occurs at the $n$-th step as the first instance in this rule B. Now it is evident that $\varepsilon_{\mathrm{B}}$ is a recurrent probabilistic event as a special case of that defined in $\S 2$. Defining $k=3$ and

$$
\begin{aligned}
& R_{1}(\mathrm{~B})=P_{0} P_{1}, \\
& R_{.2}(\mathrm{~B})=P_{0}\left(P_{2} P_{1}+P_{2}^{2}+P_{3} P_{1}\right), \\
& R_{3}(\mathrm{~B})=P_{0}\left(P_{2}+P_{3}\right)^{2}-P_{.2}^{2}\left(P_{1}+P_{2}+P_{3}\right),
\end{aligned}
$$

we have the functional equation for $n \geqq 5$,

$$
f_{\mathrm{B}}(n)=f_{\mathrm{B}}(n-1)-\sum_{j=2}^{4} R_{j-1}(\mathrm{~B}) f_{\mathrm{H}}(\boldsymbol{n}-j)
$$

with the additional conditions

(5.061) $\quad f_{\mathrm{B}}(1)=R_{1}(\mathrm{~B}) P_{\mathrm{i}}^{-1}$,

(5.062) $\quad f_{\mathrm{B}}(2)=R_{1}(\mathrm{~B})+R_{2}(\mathrm{~B}) P_{\mathrm{u}}^{-1}$,

(5.063) $\quad f_{\mathrm{B}}(3)=\left(1-f_{\mathrm{B}}(1)\right) R_{1}(\mathrm{~B})+R_{2}(\mathrm{~B})+R_{3}(\mathrm{~B}) P_{0}^{-1}$,

(5.064) $\quad f_{\mathrm{B}}(4)=\left(1-f_{\mathrm{B}}(1)-f_{\mathrm{B}}(2)\right) R_{l}(\mathrm{~B})+\left(1-f_{\mathrm{B}}(1)\right) R_{2}(\mathrm{~B})+R_{3}(\mathrm{~B})$.

Indeed the direct calculation give us $(5.061) \sim(5.064)$, in view of the relations :

(5.071) $f_{\mathrm{B}}(1)=\operatorname{Pr} .\left\{Y_{1} \in E_{1}\right\}$, 


$$
\text { (5.072) } \begin{aligned}
f_{\mathrm{B}}(2)= & \operatorname{Pr} .\left\{Y_{1} \in E_{2}\right\} \operatorname{Pr} .\left\{Y_{2} \in E_{1}\right\} \\
+ & \operatorname{Pr} .\left\{Y_{1} \in E_{2}\right\} \operatorname{Pr} .\left\{Y_{2} \in E_{1} \cup E_{2}\right\}, \\
(5.073) \quad f_{\mathrm{B}}(3)= & \operatorname{Pr} .\left\{Y_{1} \in E_{1}\right\} \operatorname{Pr} .\left\{Y_{2} \in E_{01}\right\} \operatorname{Pr} .\left\{Y_{3} \in E_{1}\right\} \\
+ & \operatorname{Pr} .\left\{Y_{1} \in E_{0}\right\} \operatorname{Pr} .\left\{Y_{2} \in E_{2}\right\} \operatorname{Pr} .\left\{Y_{3} \in E_{1} \cup E_{2}\right\} \\
+ & \operatorname{Pr} .\left\{Y_{1} \in E_{2}\right\} \operatorname{Pr} .\left\{Y_{2} \in E_{3}\right\}+\operatorname{Pr} .\left\{Y_{1} \in E_{3}\right\} \operatorname{Pr} .\left\{Y_{2} \in E_{2}\right\} \\
& \left.+\operatorname{Pr} .\left\{Y_{1} \in E_{3}\right\} \operatorname{Pr} .\left\{Y_{2} \in E_{3}\right\}\right) \operatorname{Pr} .\left\{Y_{3} \in E_{1} \cup E_{2} \cup E_{3}\right\},
\end{aligned}
$$

and similarly for $f_{B}(4)$.

For $n \geqq 5$, the occurrences of the significance at the $n$-th step for the first instance are possible exactly in one of the following 13 ways:

$\left(1^{\circ}\right)$ The first case $W_{1}(\mathrm{~B})$. This means a simultaneous occurrence of the three events: (i) $\left\{y_{i}\right\}(i=1,2, \cdots, n-2)$ is non-significant; (ii) $y_{n-1}$ $\in E_{0}$; (iii) $y_{n} \in E_{1}$.

$\left(2^{\circ}\right)$ The second case $W_{2}(\mathrm{~B})$. This means a simultaneous occurrence of the four events: (i) $\left\{y_{i}\right\}(i=1,2, \cdots, n-3)$ is non-significant; (ii) $y_{n-2}$ $\in E_{0}$; (iii) $y_{n-1} \in E_{2}$; (iv) $y_{n} \in E_{1}$.

$\left(3^{\circ}\right)$ The third case $W_{3}(\mathrm{~B})$. This means a simultaneous occurrence of the four events: (i) $\left\{y_{i}\right\}(i=1,2, \cdots, n-3)$ is non-significant; (ii) $y_{n-2}$ $\in E_{0}$; (iii) $y_{n-1} \in E_{2}$; (iv) $y_{n} \in E_{2}$.

$\left(4^{\circ}\right)$ The four case $W_{4}(\mathrm{~B})$. This means a simultaneous occurrence of the four events: (i) $\left\{y_{t}\right\}(i=1,2, \cdots, n-3)$ is non-significant; (ii) $y_{n-2} \in$ $E_{0}$; (iii) $y_{n-1} \in E_{3}$; (iv) $y_{n} \in E_{1}$.

Now let us denote each of the conditions apart from (i) of the cases $W_{1}(\mathrm{~B}), W_{:}(\mathrm{B}) . W_{3}(\mathrm{~B})$ and $W_{4}(\mathrm{~B})$ symbolically by

$$
\begin{array}{llll}
\left(1^{\circ}\right) & W_{1}(\mathrm{~B}): 01 & \left(3^{\circ}\right) & W_{3}(\mathrm{~B}): 022 \\
\left(2^{\circ}\right) & W_{3}(\mathrm{~B}): 021 & \left(4^{\circ}\right) & W_{4}(\mathrm{~B}): 031 .
\end{array}
$$

$\left(5^{\circ}\right) \sim\left(13^{\circ}\right)$ The $\nu$-th cases $(5 \leqq \nu \leqq 13)$. For each of these cases a simultaneous occurrence of the five events is assumed, where the first event is common that (i) $\left\{y_{i}\right\}(i=1,2, \cdots, n-4)$ is non-significant. The other four conditions can be expressed in our symbolical notation exactly as one of the following:

$$
\begin{aligned}
& \begin{array}{lllll}
\left(5^{\circ}\right) & 0321 & \left(8^{\circ}\right) \quad 0322 & \left(11^{\circ}\right) \quad 0323
\end{array} \\
& \begin{array}{llll}
\left(6^{\circ}\right) & 0231 & \left(9^{\circ}\right) 0232 & \left(12^{\circ}\right) 0233
\end{array} \\
& \begin{array}{llll}
\left(7^{\circ}\right) & 0331 & \left(10^{\circ}\right) 0332 & \left(13^{\circ}\right) 0333 .
\end{array}
\end{aligned}
$$

For example the case $\left(9^{\circ}\right)$ means, in addition to (i), (ii) $y_{n-3} \in E_{0}$; (iii) $y_{n-2}$ $\in E_{2}$; (iv) $y_{n-1} \in E_{3}$; (v) $y_{n} \in E_{2}$.

Now it can be readily seen that

$$
\operatorname{Pr} .\left\{W_{1}(\mathrm{~B})\right\}=\left(1-\sum_{i=0}^{n-2} f(i)\right) R_{1}(\mathrm{~B}) \text {, }
$$


(5.082) $\quad \operatorname{Pr} .\left\{W_{2}(\mathrm{~B})+W_{3}(\mathrm{~B})+W_{4}(\mathrm{~B})\right\}=\sum_{j= \pm}^{4} \operatorname{Pr} .\left\{W_{j}(\mathrm{~B})\right\}$

$$
=\left(1-\sum_{i=1}^{n-3} f_{\mathrm{B}}(i)\right) R_{2}(\mathrm{~B}) \text {, }
$$

(5.083) $\operatorname{Pr} .\left\{\sum_{j=5}^{13} W_{j}(\mathrm{~B})\right\}=\sum_{j=5}^{13} \operatorname{Pr} .\left\{W_{j}(\mathrm{~B})\right\}=\left(1-\sum_{i=0}^{n-4} f_{\mathrm{B}}(i)\right) R_{3}(\mathrm{~B})$.

Consequently we have, for $n \geqq 4$.

$$
\begin{aligned}
f_{B}(n)= & \sum_{j=1}^{13} \operatorname{Pr} .\left\{W_{j}(\mathrm{~B})\right\} \\
=\left(1-\sum_{i=0}^{n-2} f_{\mathrm{B}}(i)\right) R_{1}(\mathrm{~B}) & +\left(1-\sum_{i=0}^{n-3} f_{\mathrm{B}}(i)\right) R_{\mathbf{2}}(\mathrm{B}) \\
& +\left(1-\sum_{i=1}^{n-1} f_{\mathrm{B}}(i)\right) R_{3}(\mathrm{~B}),
\end{aligned}
$$

which will lead us to the functional equation (5.05), since we have for $n \geq 5$,

$$
\begin{aligned}
f_{\mathrm{B}}(n-1)=\left(1-\sum_{i=1}^{n-3} f_{\mathrm{B}}(i)\right) R_{1}(\mathrm{~B}) & +\left(1-\sum_{i=0}^{n-4} f_{\mathrm{B}}(i)\right) R_{2}(\mathrm{~B}) \\
& +\left(1-\sum_{i=0}^{n-5} f_{\mathrm{B}}(i)\right) R_{3}(\mathrm{~B}) .
\end{aligned}
$$

The controlling method associated with this statistical decision rule $\mathbf{B}$ may be called as the control chart method of the combined type $\mathbf{C}(1,2,3)$.

Theorem 5.1. For the statistical decision rule $\mathrm{B}$, the probability that our sequence $\left\{Y_{t}\right\}$ becomes significant is certain, that is,

$$
S(1,2,3) \equiv \sum_{n=1}^{\infty} f_{\mathbf{B}}(n)=1
$$

and more the expected values of the numbers of trials attaining their first occurrence of significance is given by

$$
\begin{aligned}
T(1,2,3) & \equiv \sum_{n=1}^{\infty} n f_{\mathrm{B}}(n) \\
& =\frac{1}{P_{1}+\frac{P_{2}^{2}}{1+P_{23}+\left(P_{23}^{2}-P_{2}^{2}\right)}+\frac{\left(P_{23}^{2}-P_{2}^{2}\right) P_{23}}{1+P_{23}+\left(P_{23}^{2}-P_{2}^{2}\right)}}
\end{aligned}
$$

where we have put

$$
P_{23}=P_{2}+P_{3}
$$

Proof: (5.12) is a special case of (2.19) given in Theorem 2.1, while (5.13) is a direct consequence of (2.20) after a rearrangement of its right-. hand terms.

The following observations on our Theorem 3.3 will be of some use in looking into its implications: 
1) The case when $P_{3}=0$, that is, $a_{2}=a_{3}$. Then our $\mathbf{C}(1,2,3)$ reduces to $\mathbf{C}(1,2)$, and $(5.12)$ becomes to be coincident with (4.12) by putting $P_{3}=0$.

2) The case when $P_{3}=0$, that is, $a_{1}=a_{2}$. Our $\mathbf{C}(1,2,3)$ reduces to a combined use of lengths 1 and 3 , and (5.12) then becomes

$$
T(1,3)=\frac{1}{P_{1}+\frac{P_{3}^{2}}{1+P_{3}+P_{3}^{2}}} .
$$

3) The case when $P_{2}=P_{3}=0$, that is, $a_{1}=a_{2}=a_{3}$. Then our $\mathbf{C}(1,2,3)$ is nothing but the usual control chart.

4) The case when $P_{1}=P_{3}=0$, that is, $a_{2}=a_{3}$, and $a_{1}=\infty$. Then our $\mathbf{C}(1,2,3)$ reduces to $\mathbf{C}(2)$, and we have

$$
T(2)=\frac{1}{\frac{P_{2}^{2}}{1+P_{2}}}=\frac{1-P_{2}^{2}}{P_{2}^{2}\left(1-P_{2}\right)} \text {. }
$$

5) The case when $P_{1}=P_{2}=0$, that is, $a_{2}=a_{1}=\infty$. Then our $\mathbf{C}(1,2.3)$ reduces to $\mathbf{C}(3)$, and we have

$$
T(3)=\frac{1}{P_{3}{ }^{2}\left(1+P_{3}+P_{3}^{2}\right)}=\frac{1-P_{3}{ }^{3}}{P_{3}^{{ }^{3}}\left(1-P_{3}\right)} .
$$

[2] Statistical controlling methods $\mathbf{C}^{(+)}(1,2,3), \mathbf{C}^{(-)}(1,2,3)$ and $\mathbf{C}^{( \pm)}(1,2,3)$. Let us define the two statistical decision rules $\mathbf{B}^{(+)}$and $\mathbf{B}^{(-)}$ similarly to the statistical decision rule $\mathrm{B}$ by replacing the sets $E_{0}, E_{1}, E_{2}$ and $E_{3}$ by $E_{0}+E_{-1}+E_{-2}+E_{-3}, E_{+1}, E_{+2}$, and $E_{+3}$ for the rule $\mathbf{B}^{(+)}$respectively and also by $E_{0}+E_{+1}+E_{+2}+E_{+3}, E_{-1}, E_{-2}$, and $E_{-3}$ for the rule $\mathbf{B}^{(-)}$ respectively. These rules $B^{(+)}$and $B^{(-)}$define the recurrent probabilistic events $\varepsilon_{\mathrm{B}(t)}$ and $\varepsilon_{\mathrm{B}(-)}$ respectively. Let us define $\varepsilon_{\mathrm{B}( \pm)}=\varepsilon_{\mathrm{B}(t)} \bigcup \varepsilon_{\mathrm{B}(-)}$, the joint recurrent event of $\varepsilon_{\mathrm{B}(+)}$ and $\varepsilon_{\mathrm{B}(-)}$, and corresponding statistical decision rule $\mathrm{B}^{( \pm)}$.

The controlling methods allied to each of these statistical decision rules $\mathbf{B}^{(+)}, \mathbf{B}^{(-)}$and $\mathbf{B}^{ \pm 1}$ will be denoted by $\mathbf{C}^{(+)}(1,2,3), \mathbf{C}^{(-)}(1,2,3)$ and $\mathbf{C}^{( \pm)}(1,2,3)$ respectively.

Let us denote by $T^{(+)}(1,2,3), T^{(-)}(1,2,3)$ and $T^{( \pm)}(1,2,3)$ the expected value of numbers of trials attaining the first occurrence of significance in each of $\varepsilon_{\mathrm{B}(+)}, \varepsilon_{\mathrm{B}(-)}$, and $\varepsilon_{\mathrm{B}( \pm)}$ respectively, while probability of our sequence $\left\{Y_{i}\right\}$ becomes significant in each of $\varepsilon_{\mathrm{B}(t)}, \varepsilon_{\mathrm{B}(-)}$ and $\varepsilon_{\mathrm{B}( \pm)}$ by $S^{(+)}(1,2,3)$, $S^{(-)}(1,2,3)$ and $S^{( \pm)}(1,2,3)$ respectively. We observe

Theorem 5.2. We have

(5.17) $S^{i-)}(1,2,3)=S^{(+)}(1,2,3)=S^{( \pm)}(1,2,3)=1$ 
(5.18) $T^{(+)}(1,2,3)=\frac{1}{P_{+1}+\frac{1}{1+P_{+33}+\left(P_{+23}^{2}-P_{+2}^{2}\right)}+\frac{\left(P_{+3}^{2}-P_{+2}^{2}\right) P_{+23}}{1+P_{+33}+\left(P_{+23}^{2}-P_{+2}^{+2}\right)}}$,

(5.19) $\quad T^{(-)}(1,2,3)=\frac{1}{P_{-1}+\frac{P_{-2^{2}}}{1+P_{-23}+\left(P_{-23}^{2}-P_{-2}^{2}\right)}+\frac{\left(P_{-23}^{2}-P_{-2}^{2}\right) P_{-23}}{1+P_{-23}+\left(P_{-23}^{2}-P_{-2}^{2}\right)}}$,

$$
T(1,2,3)=\frac{1}{\frac{1}{T^{(+)}(1,2,3)}+\frac{1}{T^{(-)}(1,2,3)}},
$$

where we have put

$$
P_{+23}=P_{+2}+P_{+3}, \quad P_{-23}=P_{-2}+P_{-3} .
$$

Proof: The proof is quite similar to that of Theorem 4.2, appealing to Theorem 4.1 and Theorem 3.1.

§ 6. Numerical results on combined uses of WEILER's control limits.

Let us consider the case when $Y_{i}=\left(X_{i 1}+X_{i 2}+\cdots+X_{i n}\right) / n-m=\bar{X}_{i}-m$, where $\left\{X_{i j}\right\}(i=1,2,3, \cdots ; j=1,2, \cdots, n)$ are a sequence of mutually independent stochastic variables each of which is normally distributed in the same distribution $N\left(m+k \sigma_{0}, \sigma_{0}{ }^{2}\right)$. Let us make use of control limits proposed by WEILER [4]:

$$
\begin{aligned}
& a_{1}=B_{1} \sigma_{0} / \sqrt{n}=3.09 \sigma_{0} / \sqrt{n}, \\
& a_{2}=B_{2} \sigma_{0} / \sqrt{n}=1.85 \sigma_{0} / v / \bar{n}, \\
& a_{3}=B_{3} \sigma_{0} / v / \bar{n}=1.26 \sigma_{0} / v / \bar{n} .
\end{aligned}
$$

Then we can write the probabilities $P_{ \pm j}(j=0,1,2,3)$. defined in $\S 5$ [3] as follows:

(6. 021) $\quad P_{+11}=\operatorname{Pr} .\left\{Y_{i} \in E_{+0}\right\}=\Phi\left(B_{3}-k \sqrt{n}\right)-\Phi(0)$,

(6.022) $P_{-0}=\operatorname{Pr} .\left\{Y_{\imath} \in E_{-0}\right\}=\Phi(0)-\Phi\left(-B_{3}-k v^{\prime} \bar{n}\right)$,

(6. 023) $\quad P_{+3}=\operatorname{Pr} .\left\{Y_{i} \in E_{+3}\right\}=\dot{\Phi}\left(B_{2}-k \sqrt{n}\right)-\Phi\left(B_{3}-k \sqrt{n}\right)$,

(6. 024) $\quad P_{-3}=\operatorname{Pr} .\left\{Y_{1} \in E_{-3}\right\}=\mathscr{D}\left(-B_{3}-k \sqrt{n}\right)-\mathscr{D}\left(-B_{2}-k \sqrt{n}\right)$,

(6.025) $\quad P_{+2}=\operatorname{Pr} .\left\{Y_{i} \in E_{+2}\right\}=\Phi\left(B_{1}-k \sqrt{n}\right)-\Phi\left(B_{2}-k_{i}^{\prime} \bar{n}\right)$,

(6.026) $\quad P_{-2}=\operatorname{Pr} .\left\{Y_{\imath} \in E_{-2}\right\}=\Phi\left(-B_{2}-k_{V} / n\right)-\Phi\left(-B_{1}-k_{V}-\bar{n}\right)$,

(6.027) $\quad P_{+1}=\operatorname{Pr} .\left\{Y_{1} \in E_{+1}\right\}=\mathscr{D}(\infty)-\mathscr{D}\left(B_{1}-k \vee \bar{n}\right)$,

(6.028) $P_{-1}=\operatorname{Pr} .\left\{Y_{1} \in E_{-1}\right\}=\Phi\left(-B_{1}-k \sqrt{ } n\right)-\Phi(-\infty)$, 
where

$$
\Phi(u)=\frac{1}{v^{\prime}-2 \pi} \int_{-\infty}^{u} \exp \left\{-t^{*} / 2\right\} d t
$$

These probabilities $P_{ \pm j}(j=0,1,2,3)$ are fundamental in calculating the expected values $T$, and are given in Table 1 . $(a)$ and $(b)$ for $n=4$ and $n=10$ respectively. The six Tables 2 are given for comparisons among the controlling procedures discussed in $\$ 4$ and $\S 5$. It is to be noted that the single uses of WEILER's control limits correspond to those ennunciated by $\mathbf{C}^{(+)}(1), \mathbf{C}^{(+)}(2)$, and $\mathbf{C}^{(+)}(3)$ while the combined ones to those by $\mathbf{C}^{(+)}(1,2)$ and $\mathbf{C}^{(+)}(1,2,3)$. We are giving the controlling methods of the types $\mathbf{C}$ and $\mathbf{C}^{( \pm)}$for the sake of comparison; these latter two types seem to be more efficient than the type $\mathbf{C}^{(+)}$for other types of alternative hypotheses, as will be discussed briefly in the following paragraph.

Table 1. (a) The fundamental probabilities $P_{ \pm f}$ $n=4, B_{1}=3.09, B_{:}=1.85 . B_{3}=1.26$

\begin{tabular}{c|c|c|c|c|c|c|c|c}
\hline$k$ & $P_{-3}$ & $P_{-2}$ & $P_{-1}$ & $P_{-0}$ & $P_{+1}$ & $P_{+1}$ & $P_{+2}$ & $P_{+3}$ \\
\hline 0.0 & 0.0717 & 0.0312 & 0.0010 & 0.3962 & 0.3962 & 0.0010 & 0.0312 & 0.0717 \\
0.2 & 0.0362 & 0.0120 & 0.0002 & 0.2961 & 0.4605 & 0.0036 & 0.0700 & 0.1214 \\
0.4 & 0.0157 & 0.0040 & 0.0001 & 0.1922 & 0.4654 & 0.0110 & 0.1358 & 0.1759 \\
0.6 & 0.0058 & 0.0011 & 0.0000 & 0.1081 & 0.4089 & 0.0294 & 0.2285 & 0.2182 \\
0.8 & 0.0018 & 0.0003 & 0.0000 & 0.0527 & 0.3121 & 0.0681 & 0.3332 & 0.2318 \\
1.0 & 0.0005 & 0.0001 & 0.0000 & 0.0222 & 0.2069 & 0.1379 & 0.4218 & 0.2107 \\
1.2 & 0.0001 & 0.0000 & 0.0000 & 0.0081 & 0.1189 & 0.2451 & 0.4637 & 0.1640 \\
1.4 & 0.0000 & 0.0000 & 0.0000 & 0.0025 & 0.0592 & 0.3859 & 0.4430 & 0.1093 \\
1.6 & 0.0000 & 0.0000 & 0.0000 & 0.0007 & 0.0255 & 0.5438 & 0.3677 & 0.0623 \\
1.8 & 0.0000 & 0.0000 & 0.0000 & 0.0002 & 0.0095 & 0.6950 & 0.2650 & 0.0304 \\
2.0 & 0.0000 & 0.0000 & 0.0000 & 0.0000 & 0.0030 & 0.8186 & 0.1656 & 0.0127 \\
\hline
\end{tabular}

Table 1. (b) The fundamental probabilities $P_{ \pm j}$ $n=10, B_{1}=3.09, B_{2}=1.85, B=1.26$

\begin{tabular}{c|c|c|c|c|c|c|c|c}
\hline$k$ & $P_{-3}$ & $P_{-2}$ & $P_{-1}$ & $P_{-1}$ & $P_{+11}$ & $P_{+1}$ & $P_{+2}$ & $P_{+3}$ \\
\hline 0.0 & 0.0717 & 0.0312 & 0.0010 & 0.3962 & 0.3962 & 0.0010 & 0.0312 & 0.0717 \\
0.2 & 0.0227 & 0.0064 & 0.0001 & 0.2344 & 0.4713 & 0.0070 & 0.1046 & 0.1534 \\
0.4 & 0.0049 & 0.0009 & 0.0000 & 0.0972 & 0.3951 & 0.0340 & 0.2453 & 0.2227 \\
0.6 & 0.0007 & 0.0001 & 0.0000 & 0.0281 & 0.2331 & 0.1164 & 0.4023 & 0.2192 \\
0.8 & 0.0001 & 0.0000 & 0.0000 & 0.0056 & 0.0963 & 0.2877 & 0.4640 & 0.1462 \\
1.0 & 0.0000 & 0.0000 & 0.0000 & 0.0008 & 0.0278 & 0.5287 & 0.3765 & 0.0662 \\
1.2 & 0.0000 & 0.0000 & 0.0000 & 0.0001 & 0.0055 & 0.7596 & 0.2145 & 0.0203 \\
1.4 & 0.0000 & 0.0000 & 0.0000 & 0.0000 & 0.0008 & 0.9094 & 0.0856 & 0.0042 \\
1.6 & 0.0000 & 0.0000 & 0.0000 & 0.0000 & 0.0001 & 0.9756 & 0.0238 & 0.0006 \\
1.8 & 0.0000 & 0.0000 & 0.0000 & 0.0000 & 0.0000 & 0.9954 & 0.0046 & 0.0001 \\
2.0 & 0.0000 & 0.0000 & 0.0000 & 0.0000 & 0.0000 & 0.9994 & 0.0006 & 0.0000 \\
\hline
\end{tabular}


Table 2.1. (a) The expected value of numbers of sample attaining the first occurrence of significance for the controlling procedure of the type C $\quad(n=4)$

\begin{tabular}{c|r|r|r|rc}
\hline$k$ & $T(1)$ & $T(2)$ & $T(3)$ & $T(1,2)$ & $T(1,2,3)$ \\
\cline { 2 - 6 } 0.0 & 499.50 & 257.33 & 111.65 & 176.77 & 87.15 \\
0.2 & 245.52 & 147.64 & 69.39 & 97.65 & 54.29 \\
0.4 & 90.41 & 50.55 & 24.90 & 35.44 & 21.81 \\
0.6 & 34.03 & 18.77 & 8.87 & 13.84 & 9.49 \\
0.8 & 14.68 & 8.69 & 3.90 & 6.60 & 5.06 \\
1.0 & 7.25 & 4.98 & 2.18 & 3.80 & 3.23 \\
1.2 & 4.08 & 3.40 & 1.50 & 2.55 & 2.35 \\
1.4 & 2.59 & 2.66 & 1.21 & 1.92 & 1.85 \\
1.6 & 1.84 & 2.30 & 1.08 & 1.56 & 1.54 \\
1.8 & 1.44 & 2.13 & 1.03 & 1.33 & 1.33 \\
2.0 & 1.22 & 2.05 & 1.01 & 1.19 & 1.19 \\
\hline
\end{tabular}

Table 2.1. (b) The expected value of numbers of sample attaining the first occurrence of significance for the controlling procedure of the type $\mathbf{C} \quad(n=10)$

\begin{tabular}{c|r|r|r|r|r}
\hline$k$ & $T(1)$ & $T(2)$ & \multicolumn{1}{|c|}{$T(3)$} & $T(1,2)$ & $T(1,2,3)$ \\
\hline 00. & 499.50 & 257.33 & 111.65 & 176.77 & 87.15 \\
0.2 & 141.14 & 80.11 & 39.25 & 54.99 & 32.25 \\
0.4 & 29.40 & 16.31 & 7.64 & 12.10 & 8.44 \\
0.6 & 8.59 & 5.64 & 2.48 & 4.31 & 3.57 \\
0.8 & 3.48 & 3.10 & 1.38 & 2.30 & 2.16 \\
1.0 & 1.89 & 2.32 & 1.09 & 1.58 & 1.56 \\
1.2 & 1.32 & 2.08 & 1.02 & 1.25 & 1.25 \\
1.4 & 1.10 & 2.02 & 1.00 & 1.09 & 1.09 \\
1.6 & 1.03 & 2.00 & 1.00 & 1.02 & 1.02 \\
1.8 & 1.00 & 2.00 & 1.00 & 1.00 & 1.00 \\
2.0 & 1.00 & 2.00 & 1.00 & 1.00 & 1.00 \\
\hline
\end{tabular}

Table 2.2. (a) The expected value of numbers of sample attaining the first occurrence of significance for the controlling procedure of the type $C^{( \pm)} \quad(n=4)$

\begin{tabular}{c|r|r|r|r|r}
\hline$k$ & $T^{( \pm)}(1)$ & $T^{( \pm)}(2)$ & $T^{( \pm)}(3)$ & $T^{( \pm)}(1,2)$ & $T^{( \pm)}(1,2,3)$ \\
\hline 0.0 & 499.50 & 499.00 & 497.81 & 257.42 & 181.03 \\
0.2 & 262.47 & 192.90 & 163.59 & 117.29 & 71.89 \\
0.4 & 90.41 & 53.13 & 42.43 & 36.60 & 23.81 \\
0.6 & 34.03 & 18.92 & 15.78 & 13.91 & 9.67 \\
0.8 & 14.68 & 8.70 & 14.39 & 6.61 & 5.07 \\
1.0 & 7.25 & 4.98 & 5.17 & 3.80 & 3.23 \\
1.2 & 4.08 & 3.40 & 3.96 & 2.55 & 2.35 \\
1.4 & 2.59 & 2.66 & 3.41 & 1.92 & 1.85 \\
1.6 & 1.84 & 2.30 & 3.16 & 1.56 & 1.54 \\
1.8 & 1.44 & 2.19 & 3.06 & 1.33 & 1.33 \\
2.0 & 1.22 & 2.05 & 3.02 & 1.19 & 1.19 \\
\hline
\end{tabular}


Table 2.2. (b) The expected value of numbers of sample attaining the first occurrence of significance for the controlling procedure of the type $\mathrm{C}^{ \pm} \quad(n=4)$

\begin{tabular}{c|r|r|r|r|r}
\hline$k$ & $T^{( \pm)}(1)$ & $T^{( \pm)}(2)$ & $T^{( \pm)}(3)$ & $T^{( \pm)}(1,2)$ & $T^{( \pm)}(1,2,3)$ \\
\hline 0.0 & 499.50 & 499.00 & 497.81 & 253.47 & 181.03 \\
0.2 & 141.14 & 88.90 & 71.62 & 58.70 & 53.43 \\
0.4 & 29.40 & 16.40 & 13.87 & 12.15 & 8.57 \\
0.6 & 8.59 & 5.64 & 5.68 & 4.31 & 3.58 \\
0.8 & 3.48 & 3.10 & 3.73 & 2.30 & 2.16 \\
1.0 & 1.89 & 232 & 3.18 & 1.58 & 1.56 \\
1.2 & 1.32 & 2.08 & 3.03 & 1.25 & 1.25 \\
1.4 & 1.10 & 2.02 & 3.00 & 1.09 & 1.09 \\
1.6 & 1.03 & 2.00 & 3.00 & 1.02 & 1.02 \\
1.8 & 1.00 & 2.00 & 3.00 & 1.00 & 1.00 \\
2.0 & 1.00 & 200 & 3.00 & 1.00 & 1.00 \\
\hline
\end{tabular}

Table 2.3, (a) The expected value of numbers of sample attaining the first occurrence of significance for the controlling procedure of the type $\mathrm{C}(+) \quad(n=4)$

\begin{tabular}{c|r|r|r|r|r}
\hline$k$ & $T^{(+)}(1)$ & $T^{(+)}(2)$ & $T^{(+)}(3)$ & $T^{(+)}(1,2)$ & $T^{(+)}(1,2.3)$ \\
\hline 0.0 & 999.00 & 998.00 & 995.63 & 514.83 & 362.06 \\
0.2 & 279.96 & 198.53 & 16654 & 122.76 & 7978 \\
0.4 & 90.82 & 53.17 & 42.44 & 36.69 & 23.85 \\
0.6 & 34.04 & 18.92 & 15.78 & 13.91 & 9.67 \\
0.8 & 14.68 & 8.70 & 14.39 & 6.61 & 5.07 \\
1.0 & 7.25 & 4.98 & 5.17 & 3.80 & 3.23 \\
1.2 & 408 & 3.40 & 3.96 & 2.55 & 2.35 \\
1.4 & 2.59 & 2.66 & 3.41 & 1.92 & 1.85 \\
1.6 & 1.84 & 2.30 & 3.16 & 1.56 & 1.54 \\
1.8 & 1.44 & 2.13 & 3.06 & 1.33 & 1.33 \\
2.0 & 1.22 & 2.05 & 3.02 & 1.19 & 1.19 \\
\hline
\end{tabular}

Table 2.3. (b) The expected value of numbers of sample attaining the first occurrence of significance for the controlling procedure of the type $\mathbf{C}(+) \quad(n=10)$

\begin{tabular}{c|r|r|r|r|r}
\hline$k$ & $T^{(+)}(1)$ & $T^{(+)}(2)$ & $T^{(+)}(3)$ & $T^{(+)}(1,2)$ & $T^{(+)}(1,2,3)$ \\
\hline 0.0 & 999.00 & 998.00 & 995.63 & 514.83 & 36206 \\
0.2 & 143.14 & 89.24 & 71.75 & 59.19 & 53.90 \\
0.4 & 29.41 & 16.40 & 13.87 & 12.15 & 8.57 \\
0.6 & 8.59 & 5.64 & 5.68 & 4.31 & 3.58 \\
0.8 & 3.48 & 3.10 & 3.73 & 2.30 & 2.16 \\
1.0 & 1.89 & 2.32 & 318 & 1.58 & 1.56 \\
1.2 & 1.32 & 2.08 & 3.03 & 1.25 & 1.25 \\
14 & 1.10 & 2.02 & 3.00 & 1.09 & 1.09 \\
1.6 & 1.03 & 2.00 & 3.00 & 1.02 & 1.02 \\
1.8 & 1.00 & 2.00 & 3.00 & 1.00 & 1.00 \\
2.0 & 1.00 & 2.00 & 3.00 & 1.00 & 1.00 \\
\hline
\end{tabular}


$\$ 7$. Examples of various statistical procedures and the powers of the allied controlling methods. Our method enunciated hither to can be applied to various statistical procedures, and in this paragraph we shall be concerned with the mutual comparisons of some simple combined uses of runs with the usual control chart method, with special reference to the $3 \sigma$-method. In view of the classical theory of testing of statistical inferences, it may be natural to consider the situation corresponding to the null hypothesis and those corresponding to the alternative ones. In what follows let us assume that our population to be sampled is distributed in a normal distribution $N\left(\xi, \sigma^{2}\right)$.

Let our null hypothesis be $H_{0}: \xi=m, \sigma^{2}=\sigma_{0}{ }^{2}$, where $m$ and $\sigma_{0}{ }^{2}$ are given numbers. On the other hand we shall be concerned with the two types of alternative ones: One is

$$
H_{\imath}: \quad \xi=m \pm k \sigma_{0}, \quad \sigma^{2}=\sigma_{0}{ }^{2}, \quad 0<k<\infty,
$$

while the other is

$$
H_{l^{*}}: \xi=m, \quad \sigma^{2}=l^{2} \sigma_{0}{ }^{2}, \quad l>1 .
$$

The expected values of numbers of numbers of trials attaining the first occurrences of recurrent probabilistic event $\varepsilon$ respectively under the hypotheses $H_{0}, H_{k}$ and $H_{l^{*}}$ will be denoted by $T_{0}, T_{k}$, and $T_{l^{*}}$ respectively. For each of the controlling methods $\mathbf{C}(1), \mathbf{C}(2), \mathbf{C}(1,2), \mathbf{C}^{( \pm)}(2)$ and $\mathbf{C}^{( \pm)}(1,2)$ these $T$ will denoted by $\left(T_{11}(1), T_{k}(1), T_{2^{*}}(1)\right),\left(T_{11}(2), T_{k}(2), T_{l^{*}}(2)\right),\left(T_{0}(1,2)\right.$, $\left.T_{k}(1,2), T_{i^{*}}(1,2)\right),\left(T_{n}^{ \pm}(2), T_{k}^{ \pm}(2), T_{i^{*}}^{ \pm}(2)\right)$ and $\left(T_{i}^{ \pm}(1,2), T_{k}^{ \pm}(1,2), T_{i^{*}}^{ \pm}(1,2)\right)$ respectively, and similarly for other methods. Our method of comparing the powers of controlling methods with each other is to compare $T_{k}^{-1}$, or $T^{-1}{ }^{*}$, according to which of alternative ones are our concerns, under the condition $T_{10}^{-1}$ are constants for all concurrent controlling methods.

Let us consider (I) the usual $3 \sigma$-method, which can be denoted by $\mathbf{C}(1)$ [3.000] in our notation, which yields us $T_{0}^{-1}=P_{1}=0.00270$. In comparison with this $3 \sigma$-method, let us now consider the following four controlling methods

$$
\begin{array}{lll}
\text { (7.03) (II) } \mathbf{C}(1,2)[3.205,2.067], & \text { (III) } \mathbf{C}^{ \pm}(1,2)[3.205,1.927], \\
& \text { (IV) } \mathbf{C}(2)[1.932], & \text { (V) } \mathbf{C}^{ \pm}(2)[1.781],
\end{array}
$$

for each of which the condition $T_{0}^{-1}=0.00270$ is nearly satisfied. It is also to be noted that each pair of two constants $a_{1}, a_{2}$ is so determined as to satisfy: $P_{1}=P_{2}^{2}\left(1+P_{2}\right)^{-1}$ for (II) and $P_{1}=2 P_{+2}\left(1+P_{+2}\right)^{-1}$ for (III) respectively, under the hypothesis $H_{0}$.

The following Tables 3.1 and 3.2 show the relative powers of these five controlling methods. 
Table 3.1. $T_{k}([X])^{-1}$ The reciprocal number of the expected value of numbers of trials for attaining the first occurrence of the event in the control method $[\mathrm{X}]$ under the hypothesis $H_{k}$

\begin{tabular}{c|c|c|c|c|c}
\hline$k$ & $(\mathrm{I})$ & $(\mathrm{II})$ & $(\mathrm{III})$ & $(\mathrm{IV})$ & $(\mathrm{V})$ \\
\hline 0.0 & 0.00270 & 0.00270 & 0.00270 & 0.00270 & 0.00270 \\
0.2 & 000324 & 0.00327 & 0.00360 & 0.00320 & 0.00362 \\
0.4 & 000500 & 0.00524 & 0.00625 & 0.00492 & 0.00667 \\
0.6 & 0.00836 & 0.00930 & 0.01176 & 0.00860 & 0.01269 \\
0.8 & 0.01398 & 0.01662 & 0.02135 & 001539 & 0.02297 \\
1.0 & 0.02278 & 0.03671 & 0.03659 & 0.02772 & 0.03786 \\
1.2 & 0.03594 & 0.04746 & 0.05921 & 0.04401 & 0.06149 \\
1.4 & 0.05480 & 0.07427 & 0.09051 & 0.06833 & 0.09147 \\
16 & 0.08076 & 0.11018 & 0.13080 & 0.10000 & 0.12837 \\
1.8 & 0.11507 & 0.15537 & 0.18439 & 0.13839 & 0.17089 \\
2.0 & 0.15866 & 0.20905 & 0.23581 & 0.18196 & 0.21692 \\
\hline
\end{tabular}

Table 3. 2. $T_{l *}([X])^{-1}$ The reciprocal number of the expected value of numbers of trials for attaining the first occurrence of the event in the control method [X] under the hypothesis $H_{l}^{*}$.

\begin{tabular}{c|c|c|c|c|c}
\hline$l$ & $(\mathrm{I})$ & $(\mathrm{II})$ & $(\mathrm{III})$ & $(\mathrm{IV})$ & (V) \\
\hline 100 & 0.00270 & 0.00270 & 0.00270 & 0.00270 & 0.00270 \\
1.25 & 0.01640 & 0.01743 & 0.01636 & 0.01329 & 0.01063 \\
1.50 & 0.04550 & 0.04835 & 0.04487 & 0.03265 & 0.02468 \\
1.75 & 0.08653 & 0.09190 & 0.08591 & 0.05725 & 0.04127 \\
2.00 & 0.13361 & 0.13982 & 0.13190 & 0.08364 & 0.05461 \\
2.25 & 0.18220 & 0.18890 & 0.17967 & 0.10959 & 0.07556 \\
2.50 & 0.23014 & 0.23602 & 0.22601 & 013420 & 0.09037 \\
2.75 & 0.27571 & 0.27958 & 0.26928 & 0.15748 & 0.10619 \\
3.00 & 0.31731 & 0.32049 & 0.31030 & 0.17765 & 0.11959 \\
\hline
\end{tabular}

The numbers in bracket in each column of these Tables show the decreasing order of the number in the same row in each Table. In view of these Tables we observe:

1) For controlling shift of population mean from $m$ to $m \pm k \sigma_{0}$ under the constant variance $\sigma^{2}=\sigma_{0}^{2}$, the method $[\mathrm{V}]=\mathbf{C}^{( \pm)}(2)[1.781]$ is the most powerful one among the five methods in our present concern until $k=1.4$, which is replaced by the method $[\mathrm{III}]=\mathbf{C}^{( \pm)}(1,2)[3.205,2.067]$ from $k=1.6$ to 2.0. There exist no reasons for adovocating these of the methods [I] and [IV].

2) For controlling change of population variance from $\sigma_{10}^{2}$ to $l^{2} \sigma_{v}{ }^{2}$, the method $[\mathrm{II}]=\mathbf{C}(1,2)[3.205,2.067]$ is definitely the most powerful among the five methods in our present concern while the methods (V) and (IV) are the two weakest. 
3) In order to control both a shift of population mean and a change of population variance, it will be advised to appeal to $\mathbf{C}^{( \pm)}(1,2)[3.205,1.927]$.

\section{KYUSHU UNIVERSITY}

\section{References}

[1] W. Feller; An introduction to probability theory and its applications. New York: John Wiley and Sons, 1950.

(2] T. KitagaWa; Successive processes of statistical inferences (1). Mem. Fac. Sci. Kyushu Univ., Ser. A, 5 (1950), 139-180.

[3] - Successive processes of statistical controls (1). Mem. Fac. Sci. Kyushu Univ, Ser. A, 7 (1952), 13-28.

[4] H. WEILER; The use of runs to control the mean in quality control. Journ. Amer. Stat. Assoc., 48 (1953), 816-825. 